\title{
LES PARASITES ET LES MALADIES DE LA TRUITE
}

\author{
Par M. H.-S. DAVIS \\ Attaché au Service des recherches aquicoles de l'U. S. Bureau of Fisheries, \\ i Washington \\ (Suite) \\ G. - pRotozoniREs. - infusorres \\ Ichthyophthirius
}

Très commun dans les étangs, l'Ichthyophthirius multifiliis éprouve rarement la Truite, car il ne peut effectuer son évolution complète dans une eau à courant rapide. Ainsi le trouve-t-on rarement installé à demeure dans les salmonicultures. Dans quelques cas, pourtant, les circonstances l'ayant occasionnellement favorisé, ce parasite a provoqué des pertes sérieuses d'alevins.

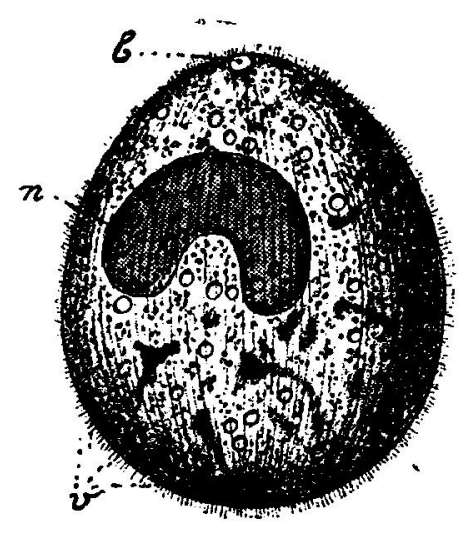

F16. 11. - Ichlhyophthirius

b) : bouche; $-n$ : noyau; $-\boldsymbol{v}$ : vacuoles contractiles. - Grossissement : environ 50 D'aprés Butschli.
Le symptôme le plus caractéristique de la maladie est l'apparition, sur le corps et les nageoires, de petites pustules d'un gris blanchâtre, à contour net. On en trouve aussi sur les branchies, mais, naturellement, moins apparentes. Les Poissons infectés se frottent contre les parois ou le fond des viviers, s'efforçant ainsi de se débarrasser de leur ennemi. Mais ce manège ne fournit pas un élément certain de diagnostic, car on l'observe aussi avec le Gyrodactylus. Lorsqu'ils sont très atteints, les Poissons perdent tout appétit et deviennent inertes.

On peut identifier l'Ichthyophthirius en grattant quelques pustules et en examinant au microscope, dans l'eau, le produit du râclage. Exceptionnellement grand pour un Protozoaire, sa longueur diamétrale atteint presque le millimètre. A l'œil $\mathrm{nu}$, on l'aperçoit sous forme de tout petits corpuscules ronds, hlanchâtres, nageant avec lenteur. Sous la loupe, il se présente avec un contour circulaire ou ovalaire et recouvert d'une multitude de cils fins, disposés en rangées, à l'aide desquelles il se meut. A la partie antérieure, il y a une petite ouverture circulaire en forme de bouche ; de nombreux granules opaques se remarquent à l'intérieur du corps ainsi que plusieurs vacuoles contractiles; vers le centre s'observe un noyau volumineux en forme de croissant. 
Le cycle vital de l'Ichthyophthirius est très complexe et intéressant; la connaissance en est indispensable pour le combattre efficacement.

Le jeune parasite est minuscule et très différent de l'adulte. Il nage acti:cment, cherchant à trouver un hôte, faute de quoi il meurt en quelques jours. Quand il est arrivé au contact d'un Poisson, il en perfore l'épiderme, s'y attachant par une des extrémités de son corps et tournant rapidement, ce qui provoque vite le déplacement de quelques cellules épithéiiales. De cette façon le jeune Infusoire se fraie peu à peu un chemin dans la profondeur de l'épiderme qui se referme derrière lui.

Ine fois inclus dans la peau on les branchies, le parasite croît rapidement et, bientôt, apparaissent, visibles à l'oeil nu, res petites pustules Hanchâtres dont il a été question plus haut. Quand il a atteint toute sa taille, l'Ichthyophthirius quitte le Poisson et tombe au fond de l'eau où il s isole bientôt par sécrétion d'une coque membraneuse. A l'intérieur de cc kyste, il se mulliplie rapidement par division donnant un nombre éven. tuellement fort élevé de jeunes individus microscopiques. Cette phase reproductrice achevée, le kyste s'ouvre donnant issue à des centaines et quelquefois des milliers de jeunes parasites qui se mettent immédiatement en quête d'un hôte.

Comme pendant la plus grande partie de son existence il vit enfermé dans l'épiderme de son hôte, soustrait à l'action des remèdes, l'Ichthyophthirius est plus difficile à combattre que les autres parasites externes. Mais quand il n'est pas tapi sous la peau, il est aisément détruit par une solution de sel ordinaire à $3 \%$ ou de sulfate d'alumine à $5 \%$ (r). Avec la première il faut prolonger le hain jusqu'à ce que le Poisson donne drs signes de détresse ; avec la seconde la durée d'immersion doit être d'une minute.

Ces opérations doivent être renouvelées plusieurs jours de suite puisque les parasites ne sont vulnérables qu'au moment où ils quittent leur hôte.

Bien entendu il faut désinfecter les viviers où a sévi l'Ichthyophthrius au moven de sel ou de chanx vive, de façon à faire périr tous les parasites pouvant exister sur le fond.

En raison de la difficulte du traitement curatif proprement dit, il est heaucoup plus pratique de placer les Poissons dans une eau à courant vif qui entraine les Infusoires devenis adulles an moment où ils abandonnent leur hôte ; ainsi sont-ils mis hors d'état de se multiplier et on rvite la réinfection. Ce procédé est d'application très aisée dans les salmonicultures. On maintient les sujets atteints dans une auge ou rigole abondamment alimentée jusqu'à ce qu'aient disparu les pustules blanches qui sont le symptôme de la maladie.

(1) En Furope on a employé aussi les bains d'eau formolén a n, ní \% fo gr. 40 par litre de solution officinale du commerce contenant fo $\%$ d'aldehydr formique). (Note du traducteur.) 


\section{Cyclochaeta}

Comme l'Ichthyophtirius, le Cyclochaeta est un Infusoire cilié, mais qui ne pénètre pas dans l'épiderme ; on Je trouve seulement à la surface du corps, sur les nageoires et sur les branchies. Habituellement, il n'abonde pas, mais, occasionnellement, pullule au point de causer un tort sérieux à son hôte. On le rencontre fréquemment en cómpagnie du Gyroiactylus et du Chilodon.

Très différent de tous les autres parasites de la Truite, Cyclochacta se laisse aisément identifier. C'est un petit organisme microscopique transparent, en forme de lentille semi-convexe, adhérant à son hôte par la partie plane. Au moyen d'une loupe simple on le distingue rampant rapidement sur le corps ou les nageoires.

L'organisation du Cyclochaeta, réellement compliquée, ne se revèle qu'au microscope sous un grossissement relativement fort. La partie ventrale, en cóntact à l'épithélium du Poisson, présente un squelette chitineux remarquable composé d'une série de plaques disposées en cercle avec prolongements vers le centre et la périphérie. En bordure, se remarque une rangée de longs cils capilliformes, à l'aide

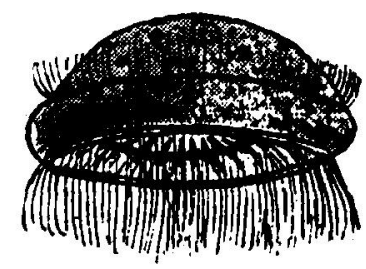

Fic. 12. - Cyclocheta.

Vu de cóté et ua pen en dessous.

Grossissement : environ 700. Diaprès Davis. desquels l'animal se déplace.

Quand l'Infusoire se présente de profil, on apercoit une seconde rangée de cils dressés en couronne bordant un sillon circulaire situé à peu près à mi-hauteur du corps et présentant en un point une invagination buccale non visible sur la figure.

Le Cyclochaeta se multiplie par scissiparité, l'animal se divisant eu deux individus semblables de même dimension. Il n'est pas certain qu'il $y$ ait une période d'enkystement et le parasite est probablement incapable de mener longtemps une existence indépendante.

Il est très facile de faire périr le Cyclochaeta; jl suffit d'un traitement par solution contenant, par litre, 30 grammes de chlorure de sodium ou 2 grammes d'acide acétique.

\section{Chilodion}

Le Chilodon est un Protozoaire qui éprouve surtout le Poisson d'étang ; on le trouve souvent en grande ahondance sur le Carassin doré ou Poisson rouge, tant jeune qu'adulte, provoquant de sévères mortalités. Il se rencontre occasionnellement chez la Truite qui, d'après Moone ( $\mathrm{r}$ ), pourrait héberger deux espèces distinctes ; dans certains cas les alevins ont été gravement éprouvés.

Le Poisson infesté ne donne aucun signe de malaise tant que le parasite

(I) Diseases of fish. - Annual report $N$. Y. Conservation Commission, XIV, pp. 83-97, 1924 . 
n'est pas très abondant; alors, il perd l'appétit et à téndance à choir sur le flanc. Quand on examine l'animal à la loupe, le Chilodon se présente sous l'aspect de menus organismes, incolores, aplatis, rampant rapidement sur les nageoires, le corps ou les branchies.

Au microscope, sous un fort grossissement, on distingue nettement son contour cordiforme, la partie antérieure du corps se terminant en pointe obtuse, tandis que la postérieure présente une échancrure. Celle-ci est due à un sillon peu profond quí s'étend, sur la face ventrale, jusqu'à la bouche ; celle-ci s'ouvre vers l'avant du corps, donnant accès à un court pharynx, à parois soutenues par un certain nombre de lamelles chitineuses. La surface du corps est couverte de cils généralement courts, mais s'allongeant sensiblement dans la région supérieure, au moven desquels l'animal se déplace. Ces cils, disposés en lignes concentriques allant d'un pôle antérieur à un pôle postérieur, donnent à l'Infusoire un aspect très caractéristique. Sa longueur est d'environ $7^{5}$ microns et sa largeur maximum de 5o microns.

Le Chilodon se reproduit par scissiparité, chaque individu se sectionnant en deur autres par constriction trans- 1 versale. Comme, en circonstances favorables, les divisions successives se suivent à intervalle le quelques heures la multiplication, est, fréquemment, d'une excessive rapidité.

Tandis que, chez les Cyprinides, le Chilodon se rencontre sur tout le corps de l'hôte, dans le cas particulier de la Truite il semble se confiner aux branchies et aux nageoires. Il n'éprouve son hôte que lorsqu'il pullule, mais, alors, peut provoquer des fortes mortalités parmi les alevins.

La destruction du Chilodon s'effectue aisé-

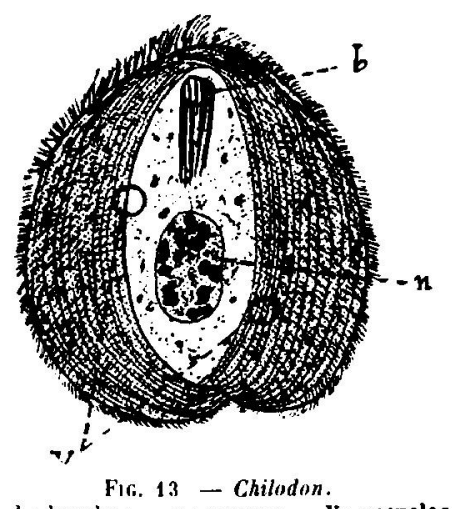

Fir. $13-$ Chilodon.
$b$ : houche: $-n$ : novau; $-v$ : vacuoles contractiles. - Grossissement: environ 500.
$D^{\prime}$ apris Davis. ment au moyen de bains dans une solution de sel commun à $3 \%$ ou d'acide acétique à $0,2 \%$. Une seule immersion suffit en général, mais, dans les cas graves, il y a lieu de réitérer à un jour d'intervalle.

Les parasites quittant volontiers leur hôte :près la inort, il convient de désinfecter aussi les auges à l'eau salée.

\section{Costia.}

Costia necatrix est un petit Flagellé (I) assex commun dans les viviers qui, dans certains cas, pullule au point d'éprouver gravement la Truite, provoquant une maladie dite " costiase " dont l'issue est rapidement fatale.

La manifestation la plus appréciable de cette affection consiste dans

(1) Les Infusoires flagellés se distinguent des Infusoirrs cilis parce qu'ils ont le corps nu et se meuvent au moyen de longs appendices ave lesquels ils fouctent l'eau. (Note du traducteur.) 
l'apparition d'un mince enduit blecâtre ou grisâtro qui s'étale sur le corps ou les nageoires. Le Poisson perd l'appétit, s'affaiblit rapidement it succombe en très peu de temps. Ces symptômes ne sont pas suffisamment caractéristiques pour assurer le diagnostic, il faut recourir à l'examen microscopique. On prélève, pour cela, une petite quantité de mucus sur la partie du corps où l'épithélium est altéré et, après l'avoir diluée dans une goutte d'eau, on l'examine à grossissement relativement fort. Le parasite se présente alors comme un menu corpuscule ovale animé de mouvements saccadés et rapides.

Quoique très petit, le Costia a une structure complexe ne se laissant définir qu'avec difficulté. Le corps est très aplati, arrondi vers l'avant et l'arrière, à régions dorsale et ventrale hien distinctes. Cette dernière est concave avec un profond sillon s'étendant obliquement du côté gauche au côté droit et plus profond à son origine où se trouve l'orifice buccal. Là prennent naissance deux paires de cils vibratiles dont l'une ne dépasse quère la longueur du corps ; l'autre est plus longue du double ou du

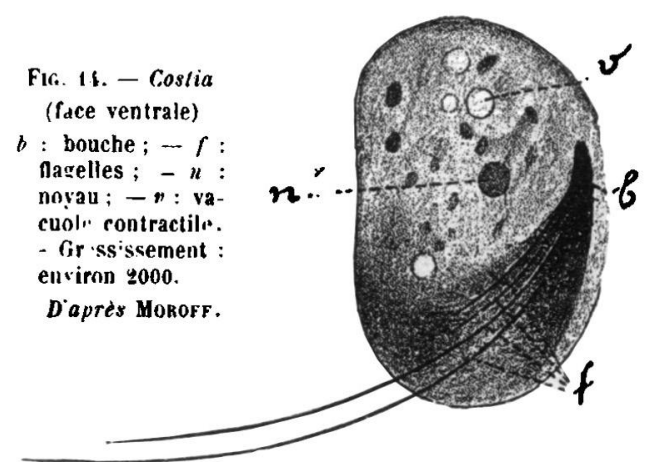
triple, les.cils étant inégaux. Ces organes servent à la nage ou à la fixation du parasite sur son hôte.

Le Costia s'installe sur la peau ou les branchies, détruisant les cellules épithéliales et se nourrissant, semble-t-il, de leurs débris.

La multiplication est irès rapide et, quand les circonstances la favorisent, le Poisson ne tarde pas à être complètement infesté.

Comme le parasite peut quitter le Poisson à volonté, au moins durant une courte période de temps, il est évident que dans les salmonicultures, où les Poissons sont à l'étroit, la maladie a toute facilité d'extension. En outre, le Costia peut aussi s'enfermer dans des kystes très résistants et mener ainsi quelque temps une vie indépendante.

A l'instar des autres parasites externes, le Costia peut être détruit par des bains salés. Mais comme il est souvent enrobé dans le mucus, il est nécessaire de donner à ces bains une durée plus forte que dans le cas du Chilodon on Cyclochoeta. La grande majorité des individus ne résiste pas à une immersion dans une solution de sel à $3 \%$ prolongée durant dix minutes. Néanmoins, il est indiqué de répéter deux ou trois fois le traitement à environ trois jours d'intervalle. On peut, aussi, recourir aux bains acidulés qui ont l'avantage d'agir plus rapidement ; une minute suffit, en effet, en utilisant une solution au $\mathrm{r} / 500^{\mathrm{e}}$ (2 grammes d'acide acétique cristallisé par litre) (I).

(A suivre).

(1) M. le Professeur Légen a préconisé des bains d'eau formolée à o.ol \% durant un quart d'heure. (Note du traducteur.) 